and instill a drop of the atropin solution after each dressing. I leave the other eye open and instill one drop of a one-quarter per cent. eserin solution in it once daily. This plan of treatment is much more comfortable to the patient than the bandaging of both eyes and the healing of the wound seems to progress as speedily as under the bandage.

\section{CASE OF CESAREAN SECTION UNDER SPINAL ANESTHESIA.}

\section{S. R. HOPKINS, M.D.} SPIINGFIELD, ILL.

A report of the following successful case is not presented because of anything particularly peculiar in the operation itself, but mainly in an attempt to show what appears to the author the superiority of spinal anesthesia over ether or chloroform in cases of Cesarean section, because of the relaxed condition of the uterine muscles likely to obtain with the patient anesthetized by either of the two latter methods.

The patient, a primipara, 28 years old, of small stature, suffered in early childhood an attack of poliomyelitis anterior acuta, as a result of which the right side of the body failed to keep pace in a matter of growth and development with the left. The right arm is $4 \frac{1}{2}$ inches shorter than the left, and the right half of the pelvis is correspondingly small, which fact, together with the markedly kyphotic condition of the lumbo-sacral spine, was largely responsible for the necessity of Cesaeran operation. The diagonal conjugate measures $9 \mathrm{~cm}$. The external conjugate (Baudelocque's diameter) measures $16 \mathrm{~cm}$.

She had been pregnant twice before, but aborted each time at about the second month. During the present pregnancy the child had never entered the true pelvis, but rode above the brim. Deeming Cesarean operation the only way possible to bring the child living into the world, the patient, on Dec. 21, 1901. was removed to the hospital two clays before full term in order to have her prepared for the operation, which preparation was done as for an ordinary celiotomy, the vagina also being rendered as nearly aseptic as possible. After labor had been in progress sufficiently long to insure good uterine contractions and dilatation of the os. there was injected into the spinal canal one-third grain of cocain hydrochlorate; the spinal fluid was used as a solving medium according to the method of Guinard of Paris, who has reported 70 cases anesthetized in this way, with none of the usual distressing post-anesthetic phenomena.

After the injection the patient was immediately transferred to the operating table, the abdomen and vagina again subjected to mechanical and chemical disinfection, by which time the entire body below the neck was anesthetic. A median incision, extending from 21/4 inches above the os pubis to $21 / 2$ inches above the umbilicus, was made through the abdominal wall and the pregnant uterus eventrated through the incision, the upper part of which was closed by Volsella forceps under the fundus, thus preventing exposure of the intestines. The uterus was at once covered by two large pads wrung out of saline solution, and in the median line between these pads an incision was made through the uterine wall, exposing the placenta attached in front. With the index finger of both hands, the placenta was torn through, the child extracted, and after its respiration was established the cord clamped and cut and placenta and membranes peeled out with the hand. During the incision of the uterus and extraction of child and membranes, the pads were several times changed for fresh hot ones and the interior of the uterus was; after being emptied, douched with hot water.

Uterine contraction was prompt, vigorous and perfect, and hemorrhage less than usual when the child is borm per vias naturales.

Interrupted sutures of heavy chromicized catgut were used to close the uterine incision, and a continuous Lembert stitch of silk to close the uterine peritoneum. By previous request of patient the tubes were doubly ligated with heavy silk in order to render her sterile. The abdominal incision was closed in the usual manner, using silk for the peritoneum, and silkworm gut for the musculature. A dry dressing was placed over all, a vaginal examination made to insure good uterine drainage, a pint of saline solution used subcutaneously below the clavicles and the patient returned to bed. The child, a female weighing $4 \frac{1}{2}$ pounds, was allowed to nurse at the end of 24 hours.

The usual annoying post-anesthetic phenomena of headache and vomiting were absent in this case, probably attributable to the use of spinal fluid as a solving medium for the cocain, thus avoiding the introduction into the spinal canal of a foreign substance (water), and also possibly because the intraspinal pressure was not increased by the injection of fluids, other than normally belonged there. In one other of my cases of spinal anesthesia wherein the cerebrospinal fluid was used to dissolve the cocain no headache nor vomiting followed the procedure.

In the case above referred to the operation required forty minutes for its completion, and anesthesia was profound during that period though tactile sense was not lost. Save for a phlebitis which developed in the left leg at the third week to disappear in five days under elevation and cold application, the mother made an uninterrupted recovery and left the hospital with her child at the end of five weeks.

The only recorded case of Cesarean section done under spinal anesthesia that I was able to find was performed by Doleris of Paris, who speaks in glowing terms of its usefulness in these cases because of the prompt and vigorous uterine contraction.

Where Cesarean section is done for placenta previa the grave danger of the failure of the uterus to contract because of the woman having suffered the loss of much blood previously is well known to all the obstetricians.

In the case of Dr. W. J. Gillette of Toledo, Ohio, wherein a Cesarean section was performed for placenta previa, after ten minutes of manipulation the uterus refused to contract, and required extirpation. It is not impossible that spinal anesthesia would have obviated this.

The well-known and powerful oxytocic properties of cocain hydrochlorate, particularly when given intraspinally, must surely recommend its use in cases wherein a failure of uterine contraction is at all possible.

Not much has been written upon the subject of intraspinal anesthesia in obstetrics. The first, and perhaps the most extensive is a monograph by Malartic, entitled, "Les injections rachidiennes de cocaine in obstetrique," Paris, 1901.

Apparently a large field is open in this direction, and cocain. subdurally for cases of uterine inertia with maternal exhaustion and in the previously mentioned class of cases, surely merits a fair trial. 